\title{
CONSUMO DE FRUTAS DE CRIANÇAS DURANTE O DISTANCIAMENTO SOCIAL NA PANDEMIA DO COVID-19 ${ }^{1}$
}

\author{
CHILDREN'S FRUIT CONSUMPTION DURING \\ SOCIAL DISTANCING IN THE PANDEMIC OF COVID-19'
}

\section{Júlia Schmitt Neves², Luciana Mello ${ }^{3}$, Malwiani Aparecida Caumo ${ }^{4}$, Natasha Gazzolla ${ }^{5}$, Franceliane Jobim Benedetti ${ }^{6}$ e Tereza Cristina Blasi ${ }^{7}$}

\section{RESUMO}

O presente estudo teve como objetivo identificar o consumo de frutas nos lanches intermediários de crianças com idade escolar, dos sete aos dez anos, durante o período de distanciamento social da COVID-19. É uma pesquisa descritiva de opinião pública realizada a partir de um questionário online elaborado no Google Forms e divulgado através das redes sociais pessoais Facebook ${ }^{\circledR}$, Instagram ${ }^{\circledR}$ e WhatsApp ${ }^{\circledR}$ dos pesquisadores por duas semanas. As perguntas referiam-se ao consumo alimentar nos lanches da manhã e da tarde. Como resultado, dos 59 participantes, o consumo de frutas nos lanches da manhã e da tarde correspondem, respectivamente, a 33 (72\%) e 50 (85\%). Dessa maneira, a inserção desses alimentos são fundamentais para o adequado crescimento e desenvolvimento infantil associado à manutenção de ótimas condições do sistema imunológico. Assim, concluiu-se que, mesmo em distanciamento social, as crianças em idade escolar avaliadas neste estudo consomem frutas nos lanches intermediários.

Palavras-chave: Dieta Saudável, Lanches, Pandemia, Nutrição da Criança.

\section{ABSTRACT}

The present study aimed to identify the consumption of fruit on the intermediary meals of schoolchildren, from seven to ten years old, during the social distance period of COVID - 19. It is a questionnaire survey of public opinion, developed on Google Forms and shared on personal social media networks like Facebook ${ }^{\circledR}$, Instagram $^{\circledR}$ and Whatsapp ${ }^{\circledR}$ from the researchers for two weeks. The questions refer to food consumption in the morning and afternoon meals. As a result, from 59 entrants, the fruit consumption in the morning and afternoon correspond, respectively to 33 (72\%) and 50 (85\%). Therefore, the inclusion of these foods is fundamental to the correct growth and childhood development associated with the maintenance of the

1 Trabalho de graduação de extensão da Disciplina Nutrição da Criança e do Adolescente. Curso de Nutrição- Universidade Franciscana - UFN.

2 Acadêmica do Curso de Nutrição e Bolsista do PET - Saúde - Interprofissionalidade - Universidade Franciscana - UFN. E-mail: juliaaschmitt@outlook.com

3 Acadêmica do Curso de Nutrição - Universidade Franciscana - UFN. E-mail: mellu.sm@gmail.com

4 Acadêmica do Curso de Nutrição e Bolsista do PET - Saúde - Interprofissionalidade - Universidade Franciscana - UFN. E-mail: malwicaumo@gmail.com

5 Acadêmica do Curso de Nutrição - Universidade Franciscana - UFN. E-mail: natashagazzolla073@gmail.com 6 Professora do Curso de Nutrição e Mestrado Materno Infantil - Universidade Franciscana - UFN. Graduada em Nutrição - UNISINOS. Mestrado e Doutorado pelo Programa de Pós-Graduação em Saúde da Criança e do Adolescente - UFRGS. E-mail: franceliane.b@gmail.com

7 Orientador - Universidade Franciscana - UFN. Professora do curso de Nutrição - UFN. Especialista em Terapia Nutricional - UFSC. Mestre em Ciências e Tecnologia de Alimentos - UFSM. Coordenadora do grupo Assistência Multidisciplinar Integrada aos Cuidadores de Pessoas com Alzheimer - AMICA. E-mail: tcb29@hotmail.com 
immune system. So, concluded that even with the social distancing, schoolchildren assessed in this study consume fruits at the intermediary meals.

\section{Keywords:}

\section{INTRODUÇÃO}

As frutas são constituintes fundamentais para uma alimentação saudável. Segundo a Organização Mundial da Saúde (OMS), o consumo insuficiente de frutas e vegetais apresenta-se como um fator de risco que pode vir a causar morte e doenças pelo mundo. Além disso, introduzir esse grupo nos hábitos alimentares, auxilia na diminuição do consumo de alimentos com excesso de energia e pobres em nutrientes (BRASIL, 2013; SICHIERI, R.; SOUZA, R. A., 2008; WHO, 2002).

A OMS recomenda o consumo diário de 400 gramas de frutas e vegetais, o que equivale a cerca de cinco porções. O Guia Alimentar para a População Brasileira salienta que a alimentação deve ter como base alimentos in natura e minimamente processados, propondo três porções de frutas diárias, uma vez que são excelentes para a manutenção da saúde e contribuem com nutrientes importantes, como fibras, minerais e vitaminas (WHO, 2003; BRASIL, 2014).

No Brasil, ainda há uma deficiência na ingestão de frutas. Isso está estritamente relacionado à condição econômica dos indivíduos. Nesse contexto, destaca-se a importância das escolas como propiciadoras do acesso das crianças a esses alimentos (JAIME, P. C.; MONTEIRO, C. A., 2005; BRASIL, 2007). Esse espaço tem como função divulgar hábitos alimentares saudáveis de forma a garantir que a Segurança Alimentar e Nutricional (SAN) ocorra de forma plena. Assim garante-se o cumprimento do direito à alimentação, estabelecendo-se a promoção de saúde através do respeito à diversidade, cultura, economia, ambiente e sociedade (BRASIL, 2006).

Para as crianças, os hábitos saudáveis relacionados à alimentação são fatores essenciais para o crescimento e desenvolvimento de modo a suprir suas necessidades de nutrientes. Do mesmo modo, dietas inadequadas tendem a prejudicar esses processos e ocasionam doenças ou agravos à saúde no futuro (CARVALHO et al., 2015). Dessa forma, incluir as frutas nos lanches intermediários das crianças demonstra ser uma estratégia eficiente de incorporação desses alimentos aos hábitos cotidianos.

A inclusão de frutas na alimentação infantil apresenta um novo desafio, pois no ano de 2020 iniciou-se um período de Distanciamento Social Ampliado (DSA), em que a sociedade permaneceu nas suas respectivas residências, restringindo o contato interpessoal (BRASIL, 2020b). Esse distanciamento ocorre devido à pandemia da COVID-19, uma doença que apresenta quadro clínico variável, de infecções assintomáticas a quadros respiratórios graves (BRASIL, 2020a). Por esse motivo, as crianças não podem ir à escola e passam o dia todo em casa podendo desencadear diferentes reflexos em relação ao comportamento e aos hábitos de vida (NCPI, 2020). 
Desta maneira, utilizar alimentos in natura e minimamente processados como base da alimentação diária é extremamente importante nesse contexto, em função da manutenção da saúde e, principalmente, da estabilidade do sistema imunológico (ASBRAN, 2020). Sendo assim, o objetivo deste trabalho foi identificar o consumo de frutas nos lanches intermediários, da manhã e da tarde, de crianças com idade escolar, dos sete aos dez anos, durante o DSA desencadeado pela COVID-19.

\section{MATERIAIS E MÉTODOS}

Este estudo baseia-se em uma pesquisa descritiva de opinião pública de caráter pontual, através da qual o participante é convidado a participar sem possibilidade de identificação (CNS, 2016).

O trabalho foi realizado a partir de um questionário online elaborado no Google Forms no mês de maio de dois mil e vinte. Ele foi disponibilizado e compartilhado através das redes sociais pessoais Facebook ${ }^{\circledR}$, Instagram ${ }^{\circledR}$ e WhatsApp ${ }^{\circledR}$ dos pesquisadores por duas semanas.

O questionário contemplava seis questões de múltipla escolha elaboradas pelos pesquisadores, questões baseadas no Guia Alimentar para a População Brasileira (BRASIL, 2014). O questionário foi direcionado para crianças em idade escolar, dos sete aos dez anos, e deveria ser preenchido pelo(s) cuidador(es). As perguntas referiam-se ao consumo alimentar nos lanches intermediários, da manhã e da tarde: Seu filho(a) consome o lanche da manhã/tarde? Qual tipo de alimento consome? Quantas frutas ele consome? Em caso positivo para o consumo de frutas, questionou-se a quantidade: uma; duas; três ou mais. Em relação aos outros alimentos havia três alternativas: pães, biscoitos ou bolos caseiros; pães, biscoitos ou bolos industrializados; leite ou iogurte.

Aos participantes que informaram seu e-mail no questionário foi enviada uma cartilha com orientações para os lanches da manhã e da tarde considerando a faixa etária da criança. A cartilha desenvolvida no programa Canva ${ }^{\circledR}$ foi elaborada com base em guias e artigos publicados pelo Ministério da Saúde (MS), Universidade Federal de Minas Gerais (UFMG) e Sociedade Brasileira de Pediatria (SBP).

Conforme Resolução no 510 de 07 de abril de 2016 (CNS, 2016), os trabalhos do tipo opinião pública com participantes não identificados não preveem o encaminhamento para o Comitê de Ética, resultando no sigilo dos participantes.

\section{RESULTADOS E DISCUSSÃO}

Durante o período de duas semanas foram obtidas 59 respostas. Os resultados demonstram que ao comparar a frequência do consumo dos lanches intermediários, nota-se que o lanche da manhã 46 (78\%) se mostra menos frequente que o lanche da tarde 59 (100\%). Na pesquisa de Mauro Fisberg et al. (2015) os resultados também apresentaram diferenças semelhantes entre a frequência 
dos lanches intermediários: o lanche da manhã (71,17\%) apresentou ser menos frequente que o lanche da tarde $(96,69 \%)$.

Em relação ao consumo de frutas nos lanches, grande parte dos que responderam ao questionário consumiam frutas. O lanche da tarde apresentou maior consumo, visto que 50 (85\%) das crianças consumiam frutas no lanche da tarde, já no lanche da manhã 33 (72\%) consumiam frutas. No que diz respeito à quantidade de frutas consumidas, os resultados estão apresentados no Gráfico 1, onde pode-se concluir que a maioria, ou seja, 37 (45\%) das crianças consomem somente uma fruta em cada lanche.

Gráfico 1 - Quantidade de frutas consumidas diariamente por crianças nos lanches.

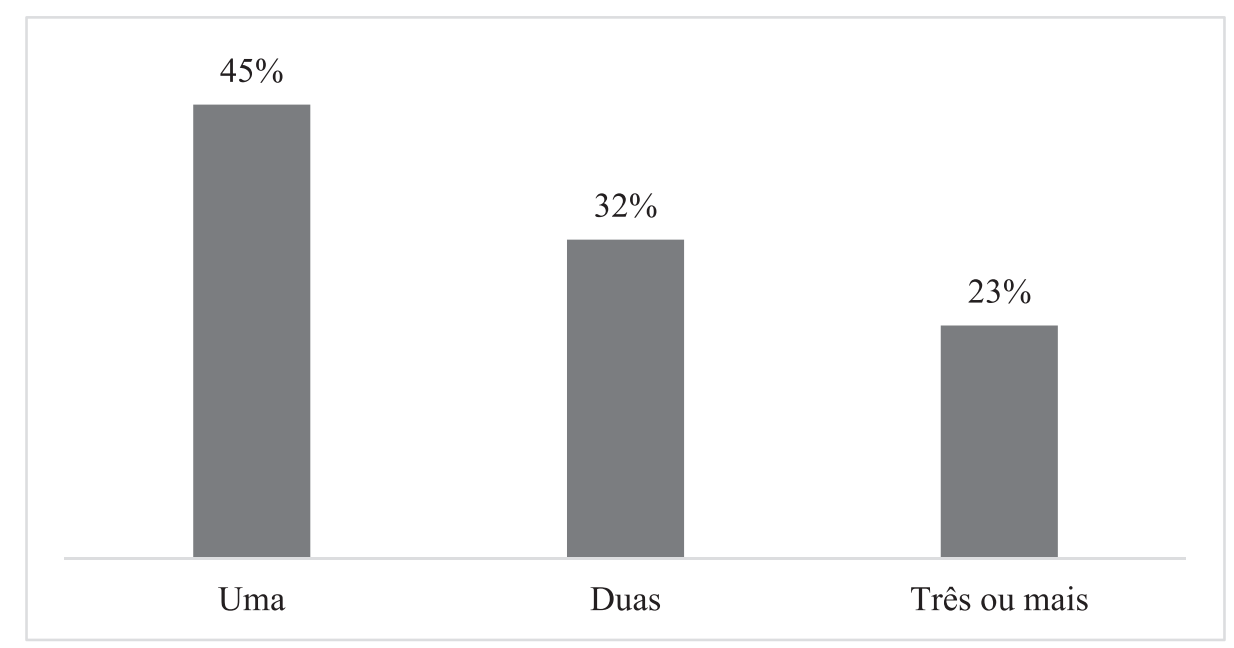

Fonte: Autoras (2020)

No lanche da manhã o consumo em quantidade de frutas foi: somente uma fruta 15 (46\%), duas frutas 10 (30\%) e três ou mais frutas 8 (24\%). Já no lanche da tarde: somente uma fruta 22 (44\%), duas frutas $17(34 \%)$ e três ou mais frutas $11(22 \%)$. Além disso, tanto no lanche da manhã quanto no lanche da tarde o consumo de frutas em quase sua totalidade foi acompanhado do consumo de outros alimentos (Gráficos 2 e 3).

No que diz respeito às crianças que consumiam os lanches, constatou-se que 13 delas (28\%) não consumiam frutas no lanche da manhã e 9 (15\%) não consumiam frutas no lanche da tarde (Gráficos 2 e 3). Observou-se também que a maioria das crianças que realizavam os lanches consumiam frutas associadas a outros alimentos, sendo 32 (54\%) e 49 (83\%) nos lanches da manhã e tarde, respectivamente.

A partir desse item, prevaleceu a associação com leite, iogurtes, pães e biscoitos caseiros, levando em consideração as crianças que consomem frutas associadas a outros alimentos 13 (41\%) de manhã e 30 (61\%) à tarde. 
Gráfico 2 - Realização do lanche da manhã e consumo de frutas.

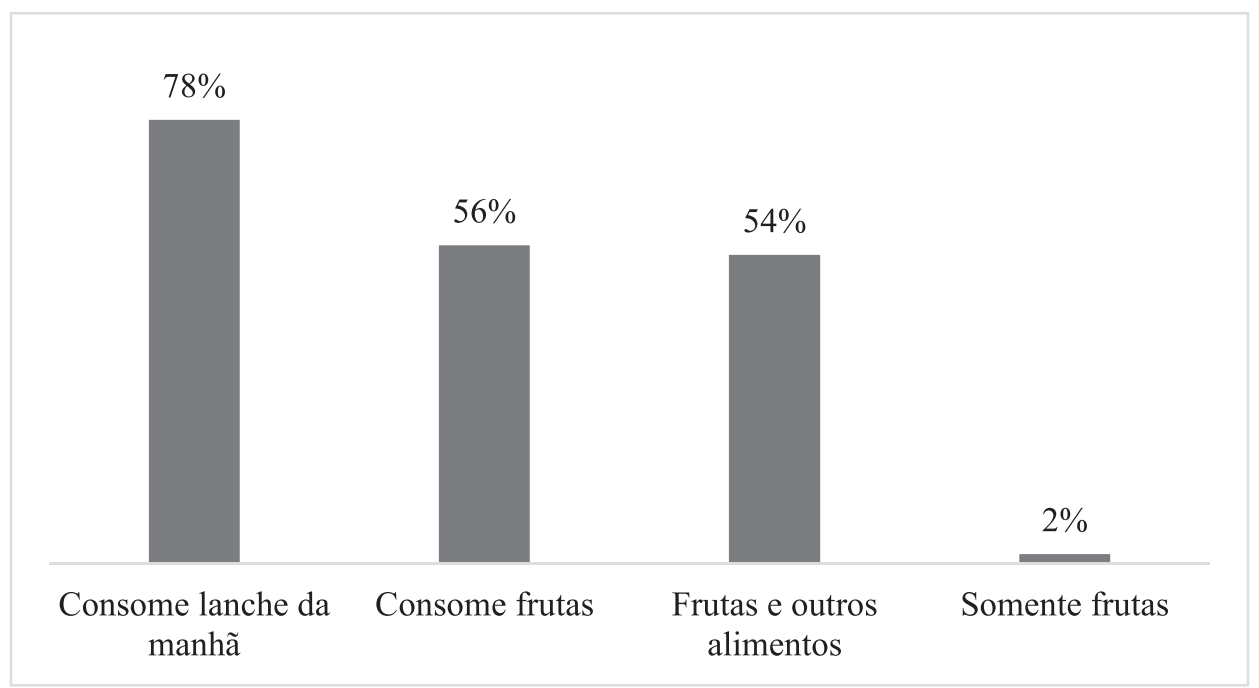

Fonte: Autoras (2020)

Gráfico 3 - Realização do lanche da tarde e consumo de frutas.

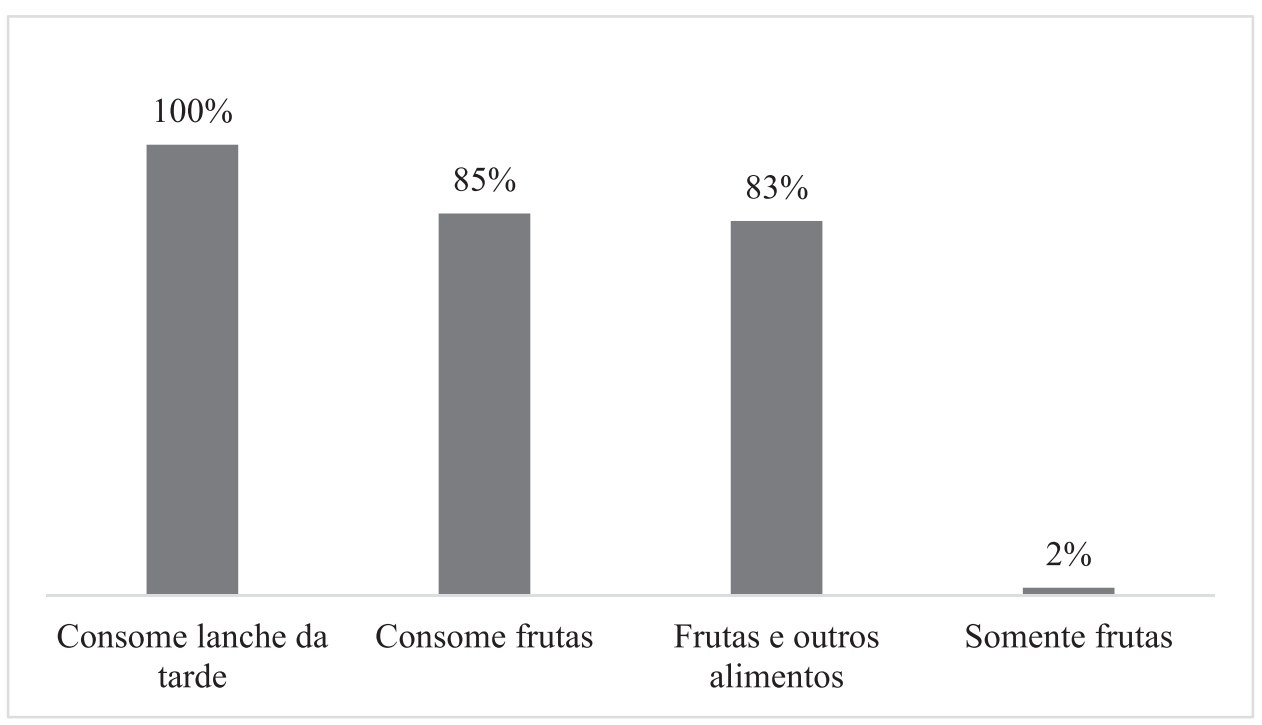

Fonte: Autoras (2020)

Alguns estudos também demonstram o não consumo de frutas pelas crianças. É o caso de uma pesquisa realizada com crianças (5 a 10 anos) e adolescentes (10 a 15 anos) em escolas públicas da região Sul do Brasil que encontrou que 12,1\% dos avaliados não consumiam frutas durante o dia (CIOCHETTO; ORLANDI; VIEIRA, 2012). Outro estudo que estimou a frequência do consumo adequado de frutas e hortaliças identificou $48,1 \%$ de inadequação no consumo de frutas (COSTA; VASCONCELOS; CORSO, 2012).

Este estudo sugere que, do total de participantes, 33 (72\%) e 50 (85\%), respectivamente, consomem frutas nos lanches da manhã e da tarde. Esses resultados divergem da análise de 380 lancheiras, compostas de 895 alimentos, que foi realizada com alunos do primeiro ano do Ensino Fundamental de escolas privadas de Santa Maria, RS, entre 2018 e 2019. A análise demonstrou 
que apenas 129 lancheiras (14\%) contemplavam frutas e sucos naturais, o que revela a baixa inserção desse grupo de alimentos nos lanches das crianças (BARBOSA, 2019).

Infere-se que o aumento no percentual do consumo de frutas observado nos dados do questionário online pode estar relacionado ao momento de distanciamento social, uma vez que há a possibilidade de as famílias estarem mais atentas à alimentação das crianças, especialmente no consumo de fontes de alimentos que auxiliam na imunidade.

Suscita-se também que os participantes que responderam à pesquisa são de classes socioeconômicas mais elevadas, pois têm acesso à internet e às redes sociais. Por outro lado, muitas crianças brasileiras necessitam diretamente da refeição fornecida pela escola para conseguir manter uma alimentação adequada e nutricionalmente balanceada. Nesses casos, o isolamento social pode impactar diretamente na qualidade dos lanches intermediários. Dessa maneira, uma alimentação saudável é primordial para manter a saúde e o sistema imunológico em ótimas condições no contexto do DSA (ASBRAN, 2020). As frutas fazem parte de um contexto alimentar saudável (BRASIL, 2014), por isso a necessidade de serem incorporadas à dieta regular de crianças em idade escolar e de serem estrategicamente inseridas nos lanches da manhã e da tarde.

Salienta-se que há características do estudo que podem se apresentar como fatores limitantes. A primeira refere-se à coleta de dados através do Google Forms. O compartilhamento do link para respostas por meio das redes sociais, não garante que os participantes contemplam todas as características alvo do estudo, ou seja, responsáveis por crianças em idade escolar. O segundo viés relaciona-se a não garantia da veracidade das informações, já que os participantes podem omitir ou responder o que acreditam ser o correto.

Por fim, destaca-se que 54 (91,5\%) dos participantes solicitaram a cartilha com orientações nutricionais para os lanches da manhã e da tarde, tornando esse compartilhamento fundamental para que haja estímulo a boas escolhas alimentares nessa faixa etária, consequentemente, auxiliando no processo de crescimento e desenvolvimento infantil. Atualmente, a geração de conteúdo nas redes sociais está em evidência, proporcionando maior disponibilidade e acesso às informações. Assim, $\mathrm{o}$ uso dessas mídias contribui para inovar métodos de Educação Alimentar e Nutricional (EAN), sendo fundamental no contexto de pandemia (WINGERT; CASTRO, 2018).

\section{CONCLUSÃO}

Os participantes consomem frutas nos lanches intermediários durante o DSA da COVID-19, sendo que a maioria consome frutas associadas a outros alimentos como leite, iogurtes, pães e biscoitos caseiros.

A imposição do DSA pode afetar o consumo alimentar de crianças, influenciando diretamente nas suas emoções e pensamentos, e isso pode acarretar escolhas alimentares inadequadas. Apesar 
disso, os dados desta pesquisa apontam que as crianças em idade escolar analisadas consomem frutas nos lanches da manhã e da tarde.

\section{REFERÊNCIAS}

AULETE, CALDAS. Novíssimo Aulete dicionário contemporâneo da língua portuguesa / Caldas Aulete; [organizador Paulo Geiger]. Rio de Janeiro: Lexikon, 2011.

ASBRAN - Associação Brasileira de Nutrição. Guia para uma alimentação saudável em tempos de COVID-19. 2020.

BARBOSA, ANA PAULA GULARTE. Jogo educativo para auxiliar a família no preparo de lancheiras saudáveis. 2019. 62f. Dissertação (Mestrado Profissional em Saúde Materno Infantil) Universidade Franciscana, Santa Maria - RS.

BRASIL. Lei no 11.346, de 15 de setembro de 2016. Cria o Sistema Nacional de Segurança Alimentar e Nutricional - SISAN com vistas em assegurar o direito humano à alimentação adequada e dá outras providências. Brasília, 15 de setembro de 2006.

BRASIL. Ministério da Saúde. Boletim Epidemiológico. Centro de Operações de Emergências em Saúde Pública - COE-nCoV; Secretaria de Vigilância em Saúde, 2020a.

BRASIL. Ministério da Saúde. Secretaria de Vigilância em Saúde. Centro de Operações Emergenciais em Saúde Pública. Doença pelo coronavírus 2019. Boletim Epidemiológico 7. 2020b.

BRASIL. Ministério da Saúde. Secretaria de Atenção à Saúde. Departamento de Atenção Básica. Política Nacional de Alimentação e Nutrição. 1. ed., 1. reimpr. Brasília: Ministério da Saúde, 2013.

BRASIL. Ministério da Saúde. Secretaria de Atenção à Saúde. Departamento de Atenção Básica. Guia alimentar para a população brasileira. 2. ed., 1. reimpr. - Brasília: Ministério da Saúde, 2014.

BRASIL. Ministério da Saúde. Secretaria de Atenção à Saúde. Departamento de Atenção Básica. Regulamentação da comercialização de alimentos em escolas no Brasil: Experiências estaduais e municipais. Brasília, 2007. 
CARVALHO, C. A. et al. Consumo alimentar e adequação nutricional em crianças brasileiras: revisão sistemática. Revista Paulista de Pediatria, v. 33, n., p. 211-221, 2015.

CIOCHETTO, C. R.; ORLANDI, S. P.; VIEIRA, M. F. A. Consumo de frutas e vegetais em escolares da rede pública no Sul do Brasil. Archivos Latinoamericanos de Nutrición, v. 62, n. 2, 2012.

CNS - CONSELHO NACIONAL DE SAÚDE. Resolução no 510, de 07 de abril de 2016. Diário Oficial da União. Edição 98, Seção 1, p. 44.

COSTA, L. C. F.; VASCONCELOS, F. A. G.; CORSO, A. C. T. Fatores associados ao consumo adequado de frutas e hortaliças em escolares de Santa Catarina, Brasil. Caderno de Saúde Pública, Rio de Janeiro, v. 28, n. 6, p. 1133-1142, jun. 2012.

FISBERG, M. et al. Hábito alimentar nos lanches intermediários de crianças pré-escolares brasileiras: estudo em amostra nacional representativa. International Journal of Neurology, v.8, n.4, p. 58-71, Set-Dez, 2015.

HINNIG, P. F.; BERGAMASCHI, D.P. Itens alimentares no consumo alimentar de crianças de 7 a 10 anos. Revista Brasileira de Epidemiologia, São Paulo, v. 15, n. 2, p. 324-34, 2012.

JAIME, P. C.; MONTEIRO, C. A. Consumo de frutas e hortaliças na população adulta brasileira, 2003. Caderno de Saúde Pública, Rio de Janeiro, 21 Sup:S19-S24, 2005.

NÚCLEO CIÊNCIA PELA INFÂNCIA. Repercussões da Pandemia de COVID-19 no Desenvolvimento Infantil, Edição Especial: 2020.

SICHIERI, R.; SOUZA, R. A. Estratégias de prevenção da obesidade em crianças e adolescentes. Caderno de Saúde Pública, Rio de Janeiro, 24 Sup 2:S209-S234, 2008.

SOCIEDADE BRASILEIRA DE PEDIATRIA. Departamento de Nutrologia. Manual de orientação para a alimentação do lactente, do pré-escolar, do escolar, do adolescente e na escola / Sociedade Brasileira de Pediatria. Departamento de Nutrologia, 3. ed. Rio de Janeiro, RJ: SBP, 2012. 
WINGERT, Karina Helena; CASTRO, Luísa Rihl. Marketing para Nutrição: conceitos e ferramentas aliados à prática da nutrição. Disciplinarum Scientia. Série: Ciências da Saúde, Santa Maria, v. 19, n. 3, p. 353-371, 2018.

WORLD HEALTH ORGANIZATION. The World Report 2002: Reducing risks, promoting healthy life. Genebra: World Health Organization, 2002.

WORLD HEALTH ORGANIZATION. Fruit and vegetable promotion initiative. Genebra: WHO, 2003. 
